\title{
A note on a possible relation between refraction and a disposition for senile nuclear cataract
}

\author{
ROBERT WEALE \\ From the Department of Visual Science, Institute of Ophthalmology, \\ Judd Street, London WC1H $9 Q S$
}

SUMMARY A study was made of records of patients who have had at least 1 senile cataract removed and for whom refraction records were available. The risk of a cataract occurring was determined as a function of precataractous refraction. Emmetropia was found to provide a sharp borderline between the low risk (about 1:1000) of hypermetropes and an increasing risk (about $1: 100$ ) for moderate myopes for patients in their early 60 s.

Fisher's interesting study on phakochronometry ${ }^{1}$ has made it likely that the formation of some types of senile cataract have a physical component attributable to ciliary stress. This is true particularly of the coronal type. Fisher ${ }^{2}$ also distinguished two maturation periods, for senile nuclear and coronal cataracts respectively, the former having the shorter period.

There are, however, some long-term mechanical considerations which may modify a disposition due to a variety of other causes. One obviously faces the question whether there is a relation between the presenile refraction of an eye and the risk of cataract in that eye. A priori we know that hypermetropic lenses tend to be latently accommodated, that is, subject to less zonular stress than is true of myopic ones. Yet apparently no study has been made to determine what relation, if any, exists between the 2 variables.

This is partly owing to the sparseness of relevant clinical data and appropriate controls. The present note is intended not to provide a solution to the problem of a possible relation between refraction and (subsequent) senile cataract but rather to draw attention to it in the hope that the compilation of relevant records may provide a valid data base. Meanwhile records for over 200 eyes were analysed in an attempt to show that such an endeavour might be fruitful.

The study necessitated a knowledge of the refraction frequency distribution of the noncataractous population for a comparable age group. Of the few sets available Brown and Kronfeld's data ${ }^{3}$ were selected for this purpose (Fig. 1A). The known

Correspondence to Professor R. Weale. statistical variations of refraction with age ${ }^{4}$ does not significantly affect the issue under discussion.

\section{Materials and methods}

The test data were obtained from the records of Moorfields Eye Hospital. Only those records were used where a cataract had been extracted at least on one side. The last refraction measurement before the operation was recorded, with its date and in addition, wherever possible, very much earlier or precataractous refractions. Only senile cataracts were considered: wherever there was a complication, such as diabetes, etc., the data were discarded. The protocol having been laid down, the records were examined by a lay assistant who was unaware of the framework of the study so that all bias could be minimised.

\section{Results}

The frequency distribution of the estimated minimum maturation periods is shown in Fig. 2. The median occurs at 3 years, and, if we bear in mind the expected skewness of the distribution, this agrees well with Fisher's estimate of 4 years for nuclear cataracts.

Fig. 1B shows the raw distribution for preoperative refractions. It is clear that there is a heavy bias on the myopic side of zero. However, as the development of nuclear cataract is accompanied by an increase in negativity, ${ }^{5}$ Fig. $1 \mathrm{~B}$ has to be corrected if the precataractous refractions are to be assessed.

The analysis of the records yielded an estimate between the preoperative refraction and the rate in dioptres per annum at which this was reached 


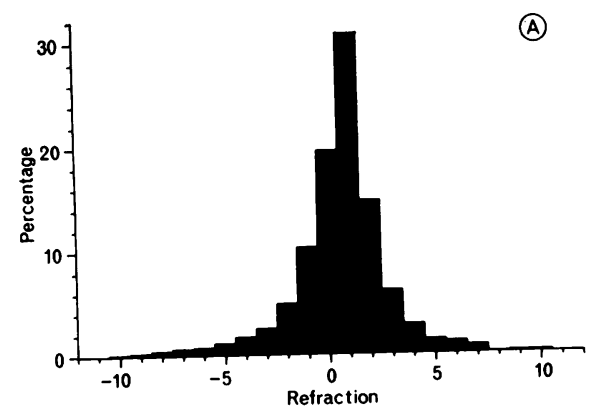

Fig. 1 Population distributions of refractive states. A. Brown and Kronfeld's data for a normal presbyopic population. B. Data for final preoperative refractions in cases of senile cataracts. C. The same after a correction for myopisation has been applied on the assumption that the maturation period is 3 years (cf. Fig. 2) and that the annual change in refraction as a function of refraction is given

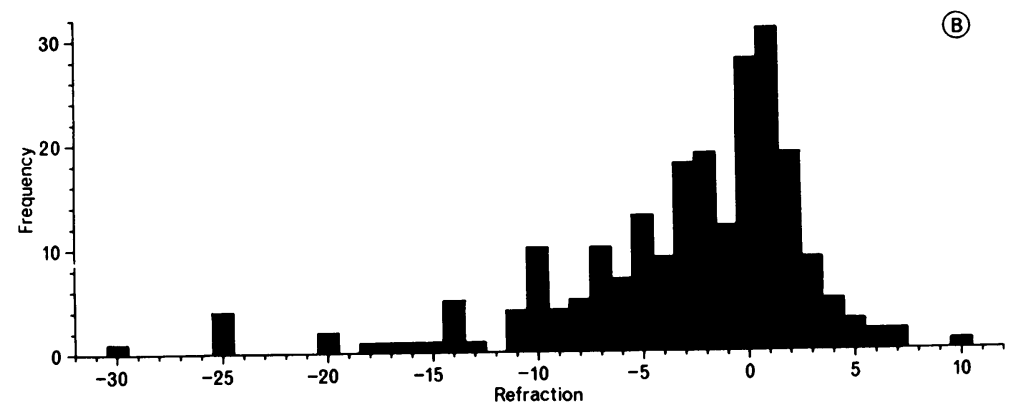
by Fig. 3.

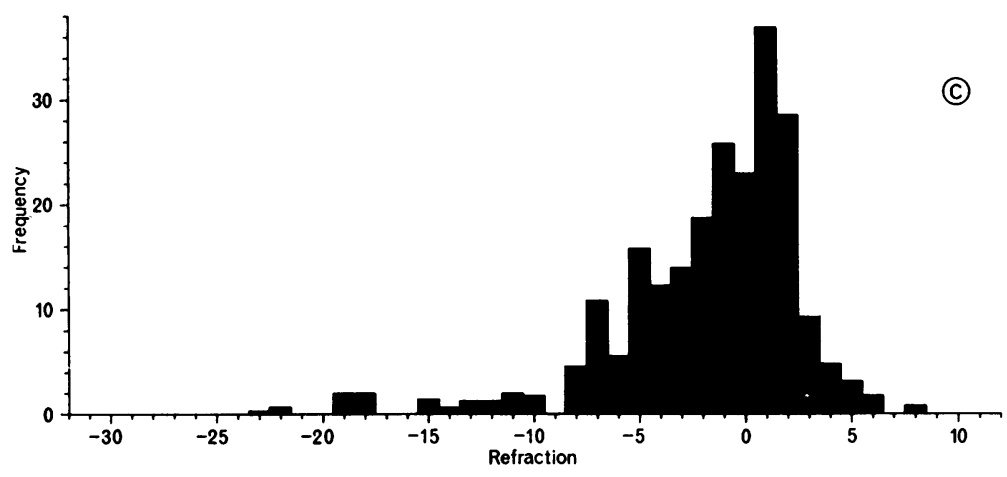

(Fig. 3). This step was needed to arrive at an estimate of the presumptive precataractous refraction (see below). The slope of the regression differed significantly from zero $(P<0.001)$ and shows that the more myopic the eye to begin with the greater the rate of development of nuclear myopia. This result is based on the difference between preoperative and precataractous refraction measurements divided by the time interval elapsing between them.

The analytical procedure was as follows. Let the fraction of the total population of a given age group having a refraction $D_{i}$ be $p\left(D_{i}\right)$ so that

$$
\sum_{a}^{m} p\left(D_{i}\right)=100 \text {. }
$$

The corresponding fraction for a coeval preoperative group is $p^{\prime}\left(D_{i}\right)$ with an analogous sum. Fig. 2 shows that the median for the presumptive maturation period is 3 years. With a statistical estimate for the annual myopisation $\Delta(D)$ obtained from the data shown in Fig. 3 the mean estimated minimum

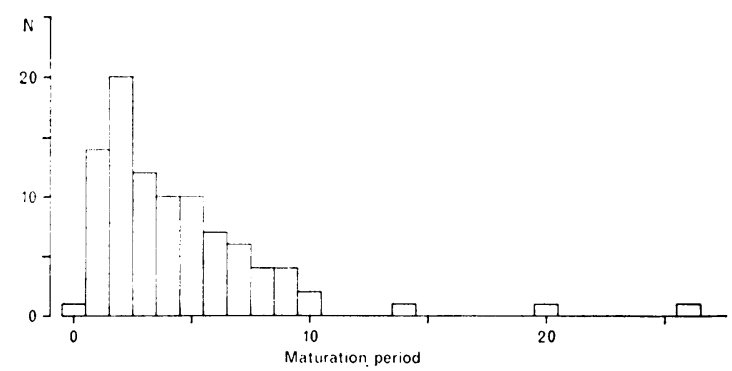

Fig. 2 Distribution of maturation periods for nuclear cataracts. 


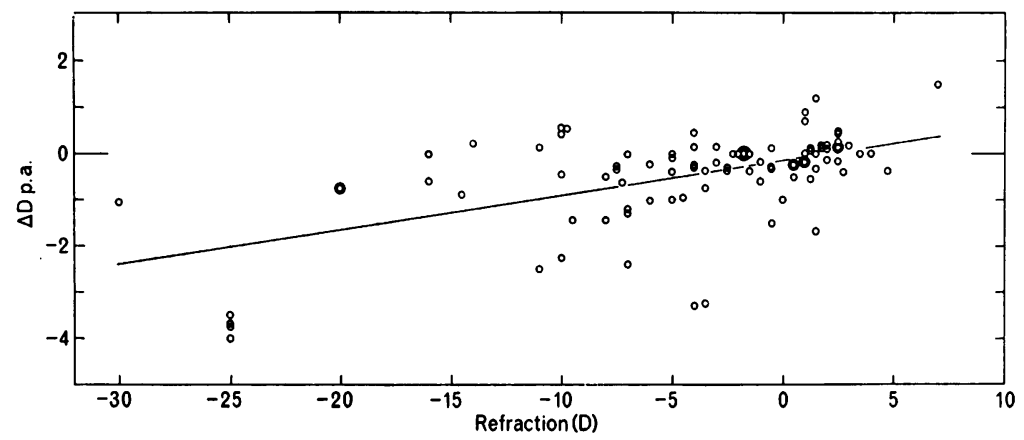

Fig. 3 Rate of change of refraction as a function of the refractive state after a cataract has been diagnosed.

change in refraction during the maturation period is $3 \Delta(\mathrm{D})$, where $\mathrm{D}$ is the preoperative refraction. The value $3 \Delta(D)$ is therefore subtracted from $D$. This gives an estimated frequency distribution for the precataractous group with percentage values of $p^{\prime \prime}\left(D_{i}\right)$.

The simplification of assuming an average maturation period of 3 years is safe, as the statistical significance of the following considerations is unimpaired even if this period is hypothetically doubled $(\mathrm{P}<0.001)$. The corrected frequency distribution is shown in Fig. 1C. The precataractous area under the resultant histogram is proportional to the total population, that is, the population that is going to suffer from cataract. Normalisation is achieved by scaling all the $P$ values by a factor so chosen as to make the area equal to that for the normal noncataractous population. It is noted that the latter may contain potential cataract patients, as it refers to a relatively young population. So far from invalidating the argument, this actually strengthens it: if, as the data suggest, myopia increases the hazard of cataract, the 'immune' population must be hypermetropic in relation to the normal distribution which has not presented.

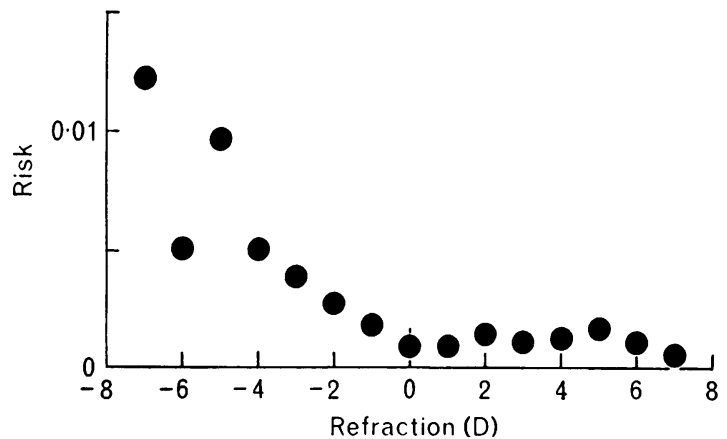

Fig. 4 The risk of a lens being extracted owing to a cataract as a function of ocular refraction. It represents the ratio of the ordinates in Figs. $1 C$ and $1 B$, and is scaled as explained in the text. $p^{\prime \prime}\left(D_{i}\right) \div p\left(D_{i}\right)$, that is, the ratio of the fraction of precataractous persons with a refraction $D_{i}$ and the fraction of 'normal' persons having the same refraction provides a measure of the risk of cataract as a function of refraction. The actual risk is obtained by multiplying this value by the incidence of senile cataract at the corresponding age obtained as set out below.

\section{Discussion}

It would seem that this is the first time a link between axial (?) and refraction myopia has been observed. This is not to suggest that there is a direct causal connection between the two, but a sequence of possibilities based on the introductory considerations cannot be ruled out.

It can be shown on the basis of 3 detailed studies of the incidence of cataract ${ }^{6-8}$ that, to a first approximation, the risk $R$ of a cataract is $1: 2500$ at the age $A$ of 50 years, and 1:10 at $A=100$ years. In general,

$$
\log \mathrm{R}+5 \cdot 8=0.048 \mathrm{~A}
$$

At the average age of the cataractous population ( $\overline{\mathrm{A}}=64.23$ years, $\sigma=9.55$ years), the risk is therefore $1: 600$. Multiplication of the aforementioned ratios by this overall risk yields the values shown in Fig. 4 .

This tells us with surprising clarity that the merest trace of myopia increases the risk: at relatively moderately small defects the risk rises 10 -fold over the minimum (normal) value. Qualitatively the result is consistent with the predictions based on laboratory tests ${ }^{9}$ and calls for an examination of the influence of other controllable parameters.

If the causal nexus is valid, then it may well become necessary to over-correct myopes, say, in their late 20 s or early 30 s so as to maintain their lenses in a slightly accommodated state. Attention would then also have to be given to the oculomotor balance.

My thanks are due to the surgeons of Moorfields Eye Hospital for allowing records of their patients to be used. 
I also thank Miss G. M. Villermet for her help, $\mathrm{Mr} \mathrm{H}$. Donovan for computational assistance, and the H. C. Binstead Memorial Fund for financial support.

\section{References}

${ }^{1}$ Fisher RF. Senile cataract: a comparative study between lens fibre stress and cuneiform opacity formation. Trans Ophthalmol Soc UK 1970; 90: 93-109.

${ }^{2}$ Fisher RF. In: The Human Lens-in relation to Cataract. Ciba Foundation Symposium No. 19 (new series). Amsterdam: Associated Scientific Publishers, 1973: 307-8.

${ }^{3}$ Brown EVL, Kronfeld PC. The refraction curve in the USA, with special reference to changes in the first two decades. 13th International Congress of Ophthalmology. Amsterdam, 1929: 1: 87-98.

'Weale RA. The Aging Eye. London: Lewis, 1963.

${ }^{5}$ Duke-Elder S, Abrams D. Ophthalmic optics and refraction. In: System of Ophthalmology. London: Kimpton, 1970: 5. ${ }^{6}$ Caird FI, Hutchinson M, Pirie A. Cataract extraction in an English population. Br J Prev Soc Med 1965; 19: 80-4.

'Halevi HS, Landau J. Hospitalized senile cataract in different Jewish communities in Israel. Br J Ophthalmol 1962; 46: 285-90.

${ }^{8}$ Lumbroso B, Melchionda C, Bacaroli I. Variations de l'âge de début de la cataracte et de l'âge moyen des opérés de cataracte. Ann Oculistique 1971; 204: 1089-108.

'Weale RA. Sex, age and the birefringence of the human crystalline lens. Exp Eye Res 1979; 29 : 449-61. 